\title{
Emission properties of a defect cavity in a two- dimensional photonic bandgap crystal slab
}

\author{
Reginald K. Lee, Oskar Painter, Benjamin Kitzke, Axel Scherer, and Amnon Yariv \\ Departments of Applied Physics and Electrical Engineering, California Institute of Technology, 1200 East California \\ Boulevard, Pasadena, California 91125
}

Received June 30, 1999; revised manuscript received December 20, 1999

\begin{abstract}
A single isolated defect within a two-dimensional photonic crystal semiconductor slab is shown to provide a lithographically tunable doubly degenerate emission resonance within the photonic bandgap, with a measured quality factor $(Q)$ of $80-150$, depending on the cavity geometry. Spontaneous emission outside the cavity linewidth is below the measurement limit of our system. Stimulated emission from this photonic crystal defect cavity is demonstrated at room temperature under pulsed optical pumping in spite of the large surface-tovolume ratio of this cavity and the associated large nonradiative surface recombination rate. (C) 2000 Optical Society of America [S0740-3224(00)01704-5]
\end{abstract}

OCIS codes: $230.3990,300.2140,230.5750,130.3130$.

\section{INTRODUCTION}

Spontaneous emission from an excited quantum state can be significantly increased or inhibited ${ }^{1}$ in a resonant cavity, depending on the relationship between the fundamental frequency of the cavity resonance and the transition frequency. Photonic bandgap (PBG) structures in lightemitting materials have been predicted to be able to exhibit enhanced or inhibited spontaneous emission. ${ }^{2,3}$ Localized electromagnetic modes can be achieved by introduction of defects within the photonic crystal lattice. These cavities have attracted considerable interest, particularly in the microwave regime, since their first observation by McCall et al. ${ }^{4}$ and by Yablonovitch et al. ${ }^{5}$ These photonic crystal defect cavities can potentially provide a means of fabricating compact nanocavity light sources, which are necessary components for the construction of high-density integrated optical circuits. ${ }^{6}$

Defects within a two-dimensional PBG crystal have been demonstrated ${ }^{7}$ in the millimeter range to be able to achieve a cavity $Q$ value of the order of $10^{4}$. Recently, Labilloy et $a l .^{8}$ observed two-dimensional light confinement in a semiconductor disk structure. The spontaneous emission properties of large hexagonal microcavity structures that use two-dimensional PBG crystals for confinement have also been previously studied. ${ }^{9,10}$ Our recent demonstration of room-temperature lasing in such structures under optical pumping was reported in Ref. 11 . Defect modes in two-dimensional PBG crystals have been studied both numerically (see, for example, Refs. 12 and 13) and experimentally in the microwave regime ${ }^{4}$; however, experimental studies of two-dimensional photonic crystal defects in the optical regime have been hampered by the nanometer-scale fabrication required for generating these structures. At the same time, they are of significant interest because of the extremely small modal volumes possible. ${ }^{14,15}$ The recent demonstration of lasing in semiconductor photonic crystal defect microcavities at low temperatures was reported in Ref. 16. In this pa- per we present an experimental study of the light emission properties of isolated defect microcavities that are embedded in a finite two-dimensional PBG crystal.

\section{DESIGN AND FABRICATION}

An oblique-view scanning electron micrograph of a typical structure is shown in Fig. 1. The microcavities consist of an isolated single defect in a two-dimensional photonic crystal lattice of air holes patterned in a hexagonal array in a freestanding thin semiconductor membrane. The membrane consists of six unstrained InGaAs quantum wells with InGaAsP barriers grown by organometallic vapor-phase epitaxy on an InP substrate with a total waveguide thickness of $150 \mathrm{~nm}$. The two-dimensional photonic lattice is patterned into the semiconductor slab by electron-beam lithography and dry etching. The patterned waveguide layer is then separated from the InP substrate to leave a freestanding membrane by use of a selective wet etch. Figure 2 is an oblique view of a cross section through a typical patterned membrane structure showing the suspended structure.

The photonic crystal defect cavity confines the light with a modal volume of $\approx 2.5(\lambda / 2 n)^{3}$, which is calculated from a finite-difference time-domain (FDTD) numerical model. ${ }^{17}$ A typical in-plane band-structure plot for this photonic lattice patterned into a thin slab, calculated by a three-dimensional FDTD technique, is shown on the left in Fig. 3. The trapezoidal solid curve in the plot is the light line, and only the slab-confined modes below the light line are shown. Figure 3 was calculated for a structure with the ratio of the hole radius to the lattice parameter, $r / a=0.35$, and the ratio of the waveguide thickness to the lattice parameter, $d / a=0.25$. This results in an in-plane bandgap, in normalized frequency units, between 0.348 and 0.465 . Numerical simulations of the defect resonance have shown ${ }^{17}$ that the defect frequency will lie near the midgap, in this case with a normalized 


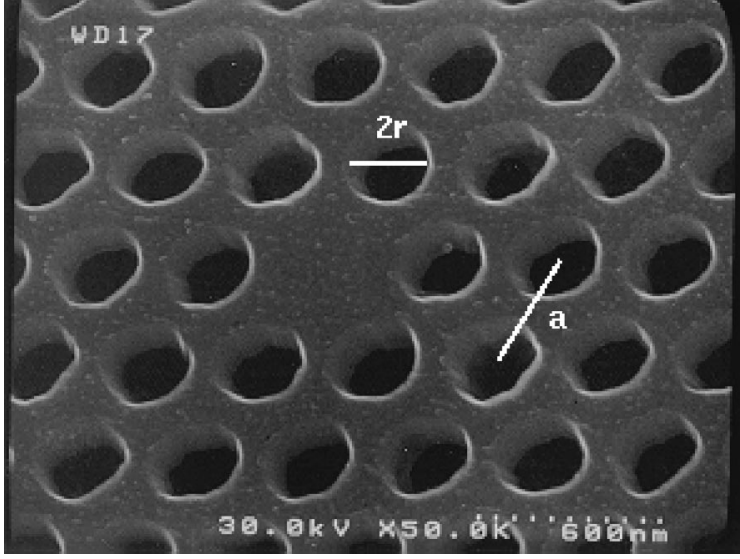

Fig. 1. Oblique-angle scanning electron micrograph image of a typical two-dimensional photonic crystal defect cavity fabricated in an InGaAsP semiconductor slab.

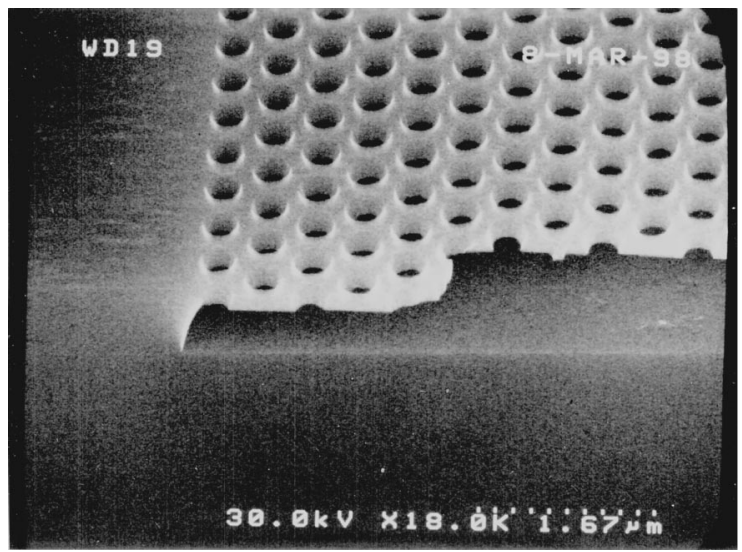

Fig. 2. Cross section through the patterned membrane structure. The InGaAsP slab in the measured devices is approximately $150 \mathrm{~nm}$ thick, and the air gap underneath the membrane can be seen.

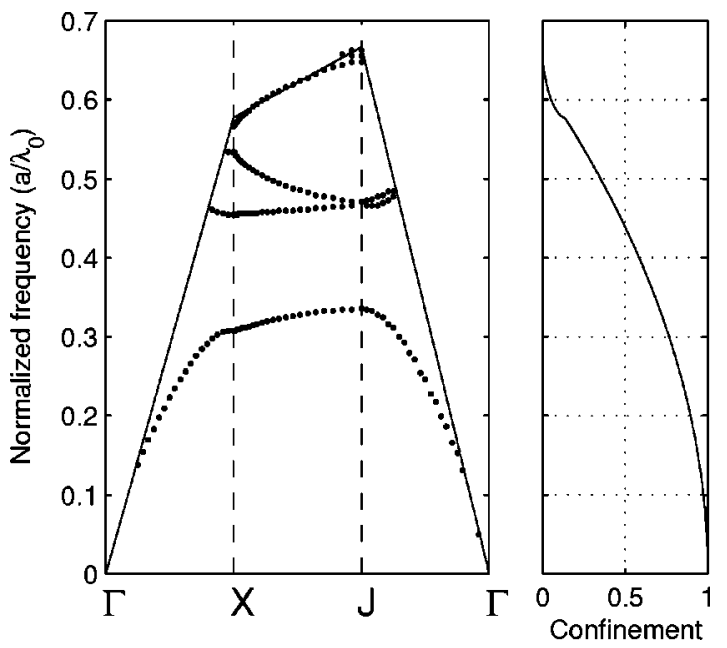

Fig. 3. Left: Calculated band structure for the twodimensional photonic crystal for the design parameters $(n d) / \lambda$ $\approx 0.33$ and $r / a \approx 0.35$. The bandgap lies in the frequency range $0.348-0.465$, with the midgap at $\approx 0.4065$. Right: Fraction of the first Brillouin zone, which lies below the light line.

frequency of 0.4065 . The total photon confinement possible in this structure depends strongly on the width of the bandgap as well as on the vertical confinement provided by the slab waveguide. At larger normalized fre- quencies a smaller portion of the Brillouin zone remains confined in the vertical direction (below the light line). This effect will be discussed in more detail in Section 3.

\section{MEASUREMENT OF DEFECT EMISSION}

The membrane microcavity was optically pumped by $10-\mathrm{ns}$ pulses at a repetition rate of $100-500 \mathrm{kHz}$ from a semiconductor laser $(\lambda=980 \mathrm{~nm})$ focused to a spot size of approximately $5 \mu \mathrm{m}$. Photoluminescence from the quantum wells at $\lambda \simeq 1.55 \mu \mathrm{m}$ was collected in the direction normal to the membrane (see inset of Fig. 6 below). The photoluminescence was passed through a monochromator and was then detected. Figure 4 shows a comparison of the photoluminescence from a semiconductor membrane patterned with the photonic crystal lattice and from an identical photonic crystal membrane containing a single defect. In both cases the PBG crystal was fabricated with a lattice spacing of $a \approx 591 \mathrm{~nm}$ and $r / a$ $\approx 0.348$. The upper plot in Fig. 4 shows that there is essentially no detectable power emitted by the photonic crystal membrane above the noise floor under these measurement conditions. Given the sensitivity in this measurement, this corresponds to a collected emission power of less than approximately $0.25 \mathrm{fW} / \mathrm{nm}$. Compared with the collected emission power from a simple unpatterned membrane, this represents a reduction in the detected spontaneous emission power by more than an order of magnitude. This decrease in emission power can be explained by three possible effects.

First, since the PBG crystal is a relatively high surfaceto-volume ratio structure, significant nonradiative recombination due to free surfaces would be expected and could dominate the total carrier recombination rate. The defect within the photonic crystal has a slightly lower surface-to-volume ratio, thereby reducing the contribu-
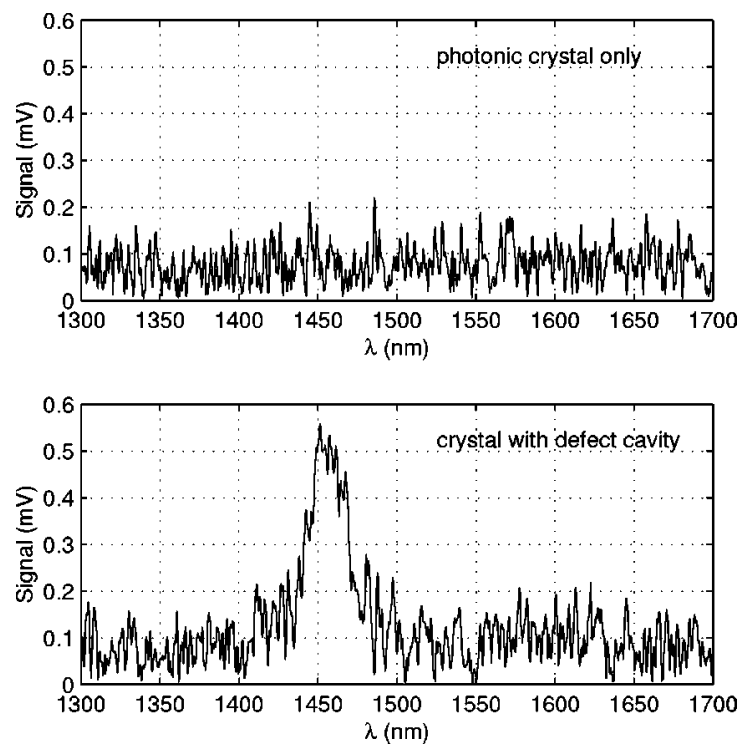

Fig. 4. Comparison of the photoluminescence spectra from a photonic crystal slab and from a slab with a single defect cavity with a lattice spacing of $a \approx 591 \mathrm{~nm}$. The top figure shows that the emission spectrum from the crystal slab has been inhibited below our measurement sensitivity limit. The lower figure shows the cavity resonance. 
tion of nonradiative surface recombination. This is a serious concern in the design of an active device, since a nonradiative dominated recombination rate would lead to low device efficiency. This topic will be discussed further in Section 4.

The second possible effect could be a change in the spatial distribution of radiation. It is important to recognize that this is a measurement not of total emission power but rather of the power emitted into the collection optics. Therefore perceived changes in the measured power could be the result of a change in the collection efficiency and not necessarily a change in the emitted power.

The third possible effect contributing to the reduced emission power could be a fundamental modification of the spontaneous emission rate, because the activematerial emission linewidth lies completely within the two-dimensional PBG. Strong modification of the spontaneous emission rate within the bandgap in this PBG slab structure has recently been shown numerically. ${ }^{18}$ When a single defect is introduced into the photonic lattice a strong emission peak is seen, as shown in the lower plot in Fig. 4. When the defect is pumped at $250 \mu \mathrm{W}$ the total integrated power detected is enhanced by a factor of more than $100 \times$ over that for the photonic crystal alone (with no defect cavity). Given the difference in surfaceto-volume ratio between the photonic crystal structure and the defect microcavity, such a large change in the total integrated emission power is not expected, assuming a constant surface recombination velocity. This result suggests that a fundamental modification of the spontaneous emission rate may be occurring. However, as previously discussed, this could also be partially attributed to a change in collection efficiency. The defect emission wavelength is centered around $\lambda=1455 \mathrm{~nm}(a / \lambda$ $=0.4061$. This defect frequency lies very close to the calculated bandgap center, $a / \lambda=0.4065$ (see Fig. 3).

The defect emission shown in Fig. 4 has a full width at half-maximum linewidth of approximately $20 \mathrm{~nm}$. This corresponds to a cavity quality factor $(Q)$ approximated as $\lambda / \Delta \lambda \approx 73$, which corresponds very well to a value of $Q$ $\approx 75$ from numerical FDTD simulations. ${ }^{17}$ For a structure containing active material, absorption losses reduce the measured $Q$ as compared with a cold cavity calculation, so linewidth is not necessarily a good measure of $Q$. A decreasing linewidth with increasing pumping is expected. However, for an active cavity below the lasing threshold, the emission linewidth can be written as follows ${ }^{19}$ :

$$
\Delta \nu_{\text {mode }}=\Delta \nu_{1 / 2}\left(1-\frac{G_{m 0}}{G_{0}}\right),
$$

where $\Delta \nu_{\text {mode }}$ is the emission mode linewidth, $\Delta \nu_{1 / 2}$ is the cold cavity linewidth, $G_{m 0}$ is a parameter proportional to the population inversion $\left[N_{2}-\left(g_{2} / g_{1}\right) N_{1}\right]$, which represents the material gain (or loss), and $G_{0}$ is a parameter proportional to the cavity losses $(\omega / Q)$. Equation (1) can be interpreted to mean that, for a cavity with absorption, $G_{m 0}<0$, the measured mode linewidth is larger than the cold cavity linewidth. The measured emission linewidth is a good measure of $Q$ only when $G_{m 0}=0$ (i.e., transparency). Furthermore, since $G_{0}$ is proportional to cavity losses, the change in linewidth with $G_{m 0}$ is less signifi- cant for low- $Q$ cavities. Conversely, for large $Q$ values, $\lambda / \Delta \lambda$ is a poorer measure of $Q$ except when $G_{m 0}$ is close to zero. Therefore we would expect that, since the measured $Q$ value for this structure is small, there should not be a large change in linewidth as a function of pumping power. This is consistent with measured linewidths, which did not change within the error of our measurements for 10-ns pulsed pumping with average powers from 200 to $500 \mu \mathrm{W}$.

Numerical simulations of the defect cavity quality factor $^{17}$ have shown that $Q$ depends on the relative position of the bandgap to the light line. Figure 3 (right) shows the fraction of the first Brillouin zone confined below the light line for the slab waveguide as a function of normalized frequency, which is related to the possible effective cavity $Q$. Measurements on defects fabricated with $r / a=0.32$ and $d / a=0.4$ result in a bandgap at slightly lower frequencies and with a calculated defect frequency of $a / \lambda=0.34$. These devices show narrower linewidths of $\Delta \lambda=7 \mathrm{~nm}$ and therefore a correspondingly larger $Q$ value of $\sim 250$, in agreement with FDTD numerical simulations. Compare this with the emission spectrum shown in Fig. 4, where a cavity $Q$ value of approximately $\lambda / \Delta \lambda=73$ was observed for a normalized defect frequency of $a / \lambda=0.41$, in qualitative agreement with the confinement behavior shown in Fig. 3.

\section{DEFECT TUNING AND STIMULATED EMISSION}

Photonic crystal defect cavities provide an inherent flexibility for control of the cavity emission through lithographic changes in the geometry. Figure 5 shows the spontaneous emission spectra for photonic crystal defect cavities fabricated with different lattice spacings from $a=960 \mathrm{~nm}$ to $a=512 \mathrm{~nm}$. The dark-gray shaded regions represent the range of $\pm 2.5 \%$ around the numerically calculated defect frequency for each structure, taking into account a small variation in $r / a$, which is due to fabrication errors. The light-gray shaded region represents the estimated band edges $( \pm 5 \%)$ from numerical simulations. The accuracy of the numerical simulations is limited by the uncertainty in the exact device parameters, which is due to fabrication errors. When the photonic crystal lattice size is tuned so that the bandgap occurs at wavelengths longer than the spontaneous emission bandwidth of the material [Fig. 5(a)], a broad spontaneous emission spectrum similar to an unpatterned sample is seen. Figures 5(b)-5(d) show cases in which the bandgap frequency is tuned to wavelengths longer than the emission bandwidth, as in Fig. 5(a). However, the spectrum is modified from the smooth spontaneous emission peak characteristic of the unpatterned material. These spectral features are caused by the modes of the photonic crystal bands (the conduction-band modes), which modify the spontaneous emission. Peaks in the spectra corresponding to the different individual conduction bands are not clearly separated in this case because the emission is angle integrated around the inplane $k=0$ point over the solid angle defined by the collection optics, with a numerical aperture of 0.65 . 
When the PBG and the defect mode frequency are well aligned with the emission bandwidth [Fig. 5(e)] a narrow emission peak can be seen at $\lambda \simeq 1.58 \mu \mathrm{m}$, with most of the emission at other wavelengths being suppressed. One can tune the defect mode frequency across the emission bandwidth while maintaining a similar cavity quality factor as well as a similar emission power. Figure 5(f) appears to have a higher signal-to-noise ratio than that of the other spectra, owing to a change in the noise bandwidth, which is due to signal averaging for this measurement. The defect mode peak in this measurement has been tuned to a shorter wavelength at $\lambda \simeq 1.46 \mu \mathrm{m}$. This represents a tuning of over $120 \mathrm{~nm}$ while a similar emission power is maintained. When the defect mode is further tuned to wavelengths shorter than the emission bandwidth no significant spectral features can be observed. Emission into the photonic bands below the bandgap (the valence-band modes) is not observed because these modes lie completely below the light line and are thus confined within the membrane plane. ${ }^{14,15}$
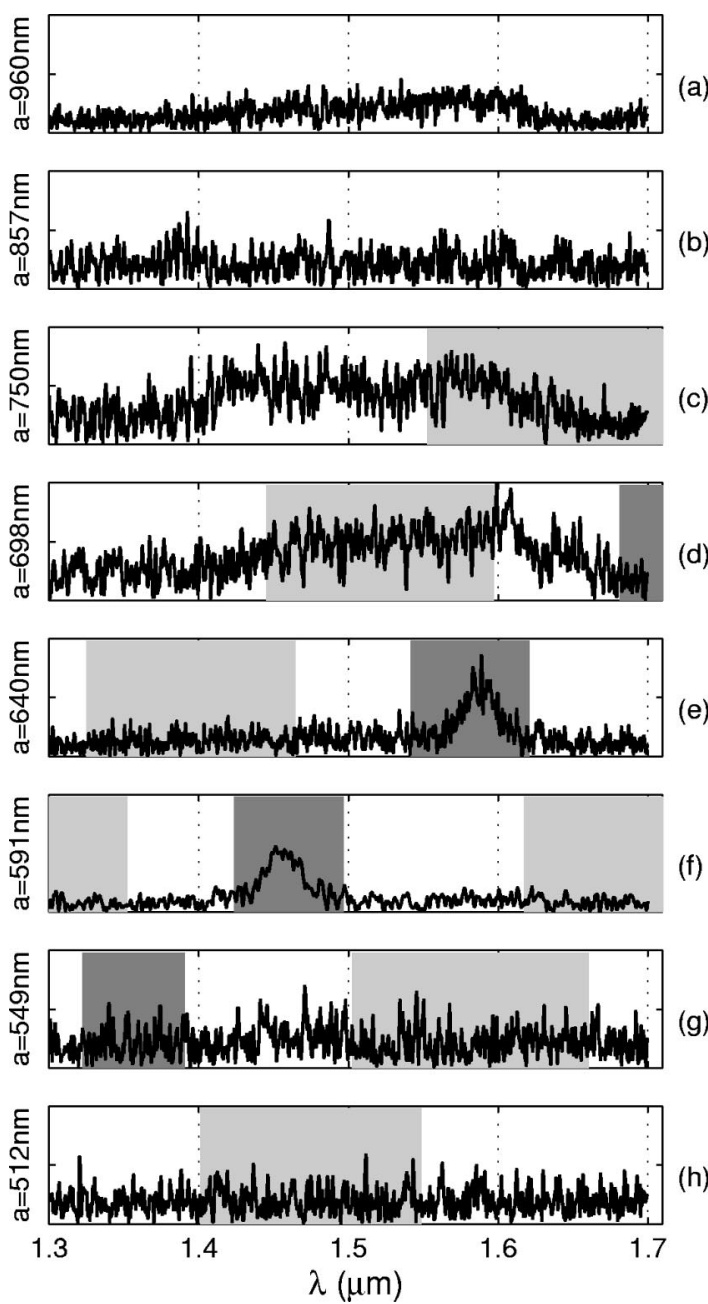

Fig. 5. Defect cavity spontaneous emission tuning. The normalized spontaneous emission spectrum for defect cavities based on photonic crystals with different lattice spacings are shown, with the lattice spacing $a$ given to the left of each plot. The dark-gray bands indicate the estimated defect frequency $a / \lambda=0.4065 \pm 1 \%$. The light-gray bands indicate the estimated band edge (with $5 \%$ error), as described in the text. In (c) and (d), only the upper (high-frequency) band edge is visible; in (g) and (h), only the lower (low-frequency) band edge is visible.

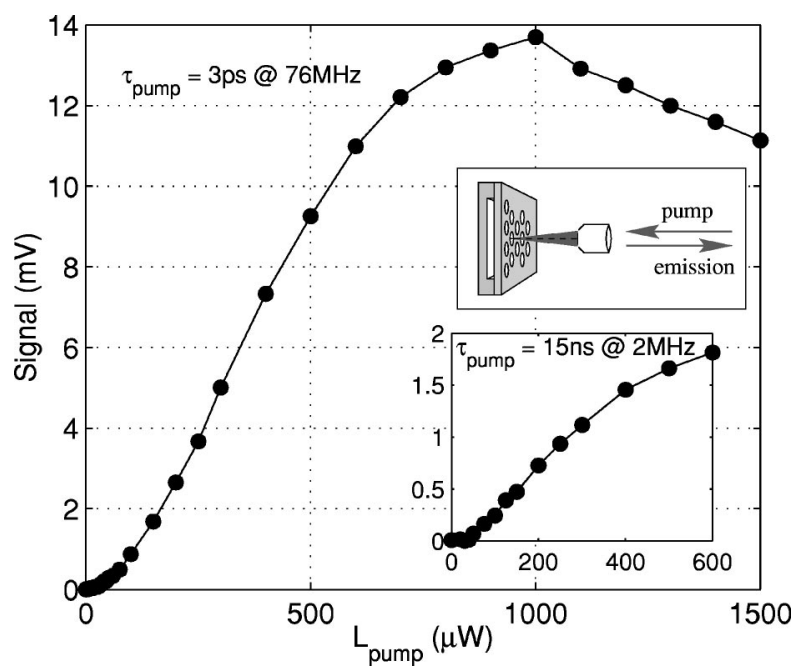

Fig. 6. Defect cavity light output versus average pump power plot. The two inset figures show the pumping and detection configuration (top) and a similar $L-L$ plot for longer pumping pulses (bottom). The light output shows a clear change in slope efficiency, indicating the onset of stimulated emission at approximately $P_{\text {avg }}=30 \mu \mathrm{W}$. Above $1 \mathrm{~mW}$, the roll-off in the radiative efficiency can be seen as the device begins to overheat.

The light output from the defect microcavity versus pumping power $(L-L)$ is displayed in Fig. 6, showing a gradual change in the slope at $P_{\text {avg }} \approx 30 \mu \mathrm{W}$. A change in the radiative efficiency, owing to the onset of stimulated emission, could explain this effect. At approximately $750 \mu \mathrm{W}$ the slope efficiency gradually decreases until $1000 \mu \mathrm{W}$, where the output power actually begins to decrease. This reduction in radiative efficiency is attributed to the increased active-layer temperature and carrier density. The higher temperature increases the point defect and the surface nonradiative recombination because of the increased carrier diffusion. In addition, and probably more importantly, the larger carrier density increases the contribution of Auger recombination, which increases as the cube of the carrier density as well as increasing exponentially with temperature. Figure 6 (lower inset) shows the light output measurement taken with 15-ns pulses, which shows a similar behavior. This indicates that thermal heating differences are not significant, since the pumping occurs faster than the structure thermal response time. The change in slope can also be due to the saturation of nonradiative centers; however, this can be verified by comparing $L-L$ plots for different spectral slices. Around the cavity resonance a response essentially identical to that shown in Fig. 6 is seen. However, if the cavity resonance is spectrally filtered and the light output away from the cavity response is measured, the output is linearly proportional to the pump, and no change in slope is seen.

\section{CONCLUSION}

We have demonstrated narrow-linewidth emission from an isolated defect in a two-dimensional photonic bandgap crystal in the InP material system. This type of cavity has a small modal volume ${ }^{17}$ of $\approx 2.5(\lambda / 2 n)^{3}$. To our knowledge, this is one of the smallest modal volume ac- 
tive semiconductor cavities in the near-infrared ever measured. The effect of the photonic crystal defect results in a narrow spontaneous emission peak corresponding to the cavity response. Stimulated emission into the defect mode is demonstrated at room temperature under pulsed optical pumping conditions, and the mode frequency is tuned lithographically across the material emission bandwidth.

\section{ACKNOWLEDGMENTS}

The authors thank Jeffrey Ungar and Se Oh, who are with Ortel Corporation, for supplying the material grown for this paper. This research was supported by the Army Research Office, the National Science Foundation, and the Office of Naval Research. R. K. Lee's work and O. Painter's work are both supported by the National Science and Engineering Research Council of Canada.

R. K. Lee can be reached by e-mail at leereg@its.caltech.edu.

\section{REFERENCES}

1. E. Purcell, "Spontaneous emission probabilities at radio frequencies," Phys. Rev. 69, 681 (1946).

2. E. Yablonovitch, "Inhibited spontaneous emission in solidstate physics and electronics," Phys. Rev. Lett. 58, 20592062 (1987).

3. S. John, "Strong localization of photons in certain disordered dielectric superlattices," Phys. Rev. Lett. 58, 24862489 (1987).

4. S. McCall, P. Platzman, R. Dalichaouch, D. Smith, and S. Schultz, "Microwave propagation in two-dimensional dielectric lattices," Phys. Rev. Lett. 67, 2017-2020 (1991).

5. E. Yablonovitch, T. Gmitter, R. Meade, A. Rappe, K. Brommer, and J. Joannopoulos, "Donor and acceptor modes in photonic band-structure," Phys. Rev. Lett. 67, 3380-3383 (1991).

6. T. Baba, "Photonic crystals and microdisk cavities based on
GaInAsP-InP system,” IEEE J. Sel. Top. Quantum Electron. 3, 808-830 (1997).

7. S. Lin, V. Hietala, S. Lyo, and A. Zaslavsky, "Photonic band gap quantum well and quantum box structures: a high-Q resonant cavity," Appl. Phys. Lett. 68, 3233-3235 (1996).

8. D. Labilloy, H. Benisty, C. Weisbuch, T. Krauss, C. Smith, R. Houdre, and U. Oesterle, "High-finesse disk microcavity based on a circular Bragg reflector," Appl. Phys. Lett. 73, 1314-1316 (1998).

9. R. Lee, O. Painter, B. D'Urso, A. Scherer, and A. Yariv, "Measurement of spontaneous emission from a twodimensional photonic band gap defined microcavity at nearinfrared wavelengths," Appl. Phys. Lett. 74, 1522-1524 (1999).

10. C. Smith, H. Benisty, D. Labilloy, U. Oesterle, R. Houdre, T. Krauss, R. De la Rue, and C. Weisbuch, "Near-infrared microcavities confined by two-dimensional photonic bandgap crystals," Electron. Lett. 35, 228-230 (1999).

11. R. Lee, O. Painter, B. Kitzke, A. Scherer, and A. Yariv, "Photonic bandgap disk laser," Electron. Lett. 35, 569-570 (1999).

12. N. Kawai, M. Wada, and K. Sakoda, "Numerical analysis of localized defect modes in a photonic crystal: twodimensional triangular lattice with square rods," Jpn. J. Appl. Phys., 37, 4644-4647 (1998), Pt. 1.

13. T. Ueta, K. Ohtaka, N. Kawai, and K. Sakoda, "Limits on quality factors of localized defect modes in photonic crystals due to dielectric loss," J. Appl. Phys. 84, 6299-6304 (1998).

14. R. Coccioli, M. Boroditsky, K. Kim, Y. Rahmat-Samii, and E. Yablonovitch, "Smallest possible electromagnetic mode volume in a dielectric cavity," IEE Proc.: Optoelectron. 145, 391-397 (1998).

15. P. Villeneuve, S. Fan, S. Johnson, and J. Joannopoulos, "Three-dimensional photon confinement in photonic crystals of low-dimensional periodicity," IEE Proc.: Optoelectron. 145, 384-390 (1998).

16. O. Painter, R. Lee, A. Scherer, A. Yariv, J. O’Brien, P. Dapkus, and I. Kim, "Two-dimensional photonic band-gap defect mode laser," Science 284, 1819-1821 (1999).

17. O. Painter, J. Vuckovic, and A. Scherer, "Defect modes of a two-dimensional photonic crystal in an optically thin dielectric slab," J. Opt. Soc. Am. B 16, 275-285 (1999).

18. J. Hwang, H. Ryu, and Y. Lee, "Spontaneous emission rate of an electric dipole in a general microcavity," Phys. Rev. B 60, 4688-4695 (1999).

19. See, for example, A. Yariv, Quantum Electronics (Wiley, New York, 1989). 